\title{
Troponin I as a mortality marker after lung resection surgery - a prospective cohort study.
}

\section{CURRENT STATUS: UNDER REVIEW}

BMC Anesthesiology $\triangle B M C$ series

\section{Ricardo Uchoa}

Universidade de Sao Paulo Instituto do Coracao

Bruno Caramelli

Universidade de Sao Paulo

bcaramel@usp.brCorresponding Author

ORCiD: https://orcid.org/0000-0001-6718-8456

\section{DOI:}

10.21203/rs.2.21368/v1

\section{SUBJECT AREAS}

Anesthesiology \& Pain Medicine

\section{KEYWORDS}

Troponin, non-cardiac surgery, thoracic surgery, cardiovascular complications, cardiovascular risk, perioperative care. 
Abstract

Background: Cardiovascular complications associated with thoracic surgery increase morbidity, mortality, and treatment costs. Elevated cardiac troponin level represents a predictor of complications after non-cardiac surgeries, but its role after thoracic surgeries remains undetermined. The objective of this study was to analyze the relationship between troponin I elevation and morbidity and mortality after one year in patients undergoing lung resection surgery. Methods: This prospective cohort study evaluated 151 consecutive patients subjected to elective lung resection procedures using conventional and video-assisted thoracoscopic techniques at a University Hospital in Brazil, from July 2012 to November 2015. Preoperative risk stratification was performed using the scores obtained by the American College of Physicians (ACP) and the Society of Cardiology of the state of São Paulo (EMAPO) scoring systems. Troponin I levels were measured in the immediate postoperative period (POi) and on the first and second postoperative days. Results : Most patients had a low risk for complications according to the ACP (96.7\%) and EMAPO (82.8\%) scores. Approximately $49 \%$ of the patients exhibited increased troponin I ( $\geq 0.16 \mathrm{ng} / \mathrm{ml})$, at least once. Multivariate analysis showed that the elevation of troponin I, on the first postoperative day, correlated with a 12 -fold increase in mortality risk within one year ( $\mathrm{HR} 12.02,95 \% \mathrm{Cl}: 1.82-79.5 ; \mathrm{p}=0.01)$. Conclusions : In patients undergoing lung resection surgery, with a low risk of complications according to the preoperative evaluation scores, an increase in troponin I levels above $0.16 \mathrm{ng} / \mathrm{ml}$ in the first postoperative period correlated with an increase in mortality within one year. Keywords: Troponin, non-cardiac surgery, thoracic surgery, cardiovascular complications, cardiovascular risk, perioperative care.

\section{Full Text}

Due to technical limitations, full-text HTML conversion of this manuscript could not be completed. However, the manuscript can be downloaded and accessed as a PDF.

\section{Figures}

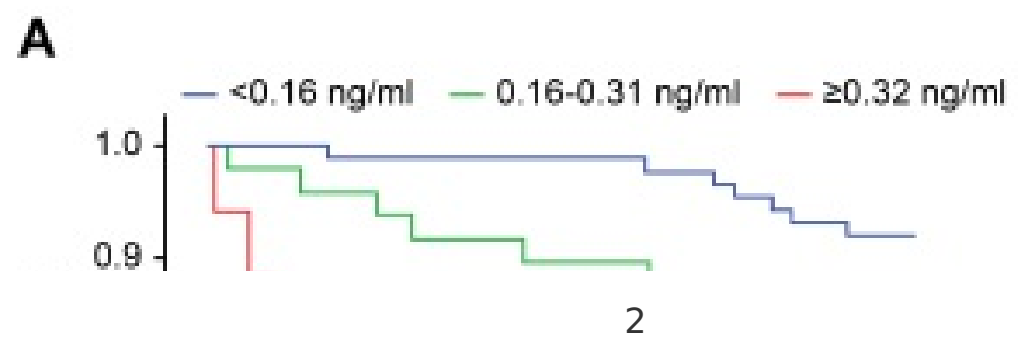




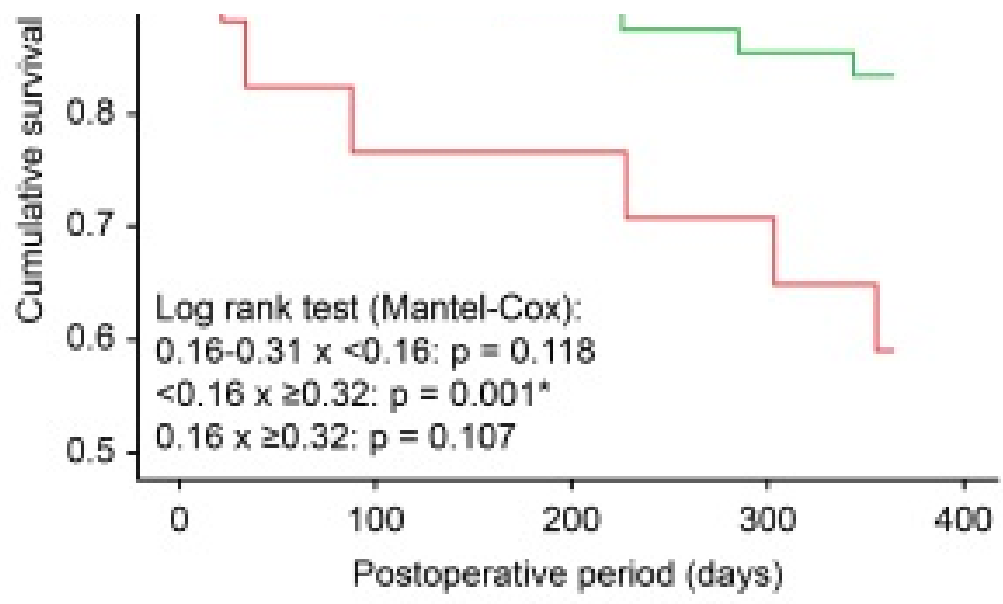

B

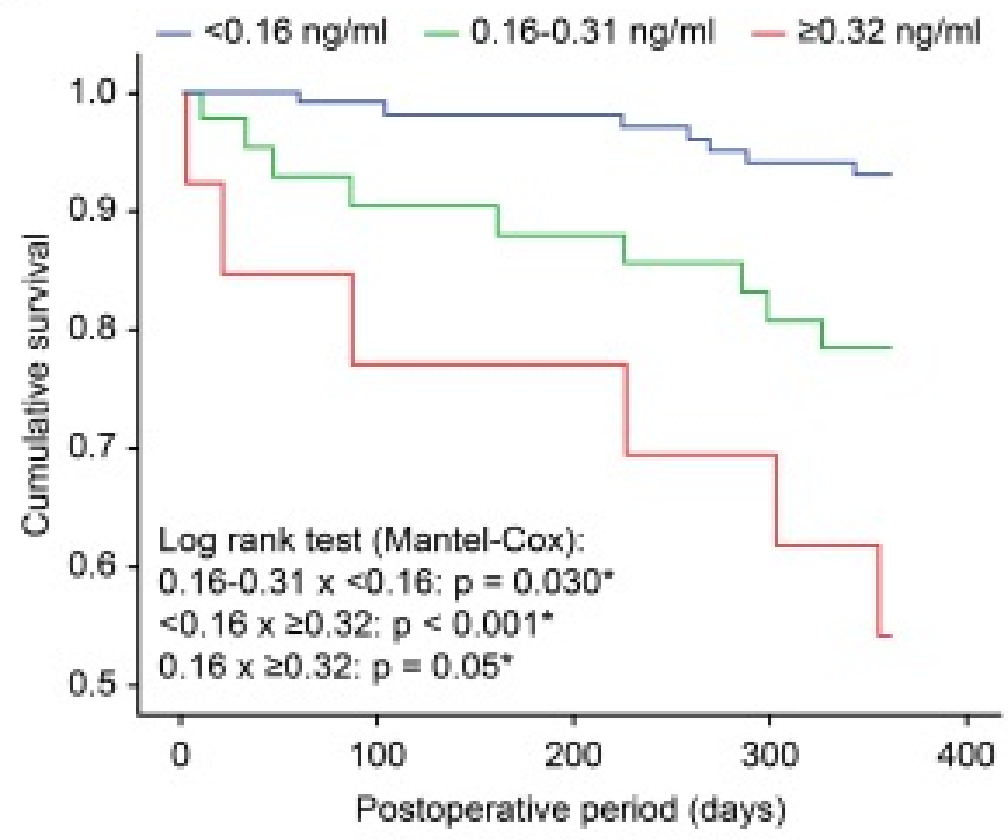

C

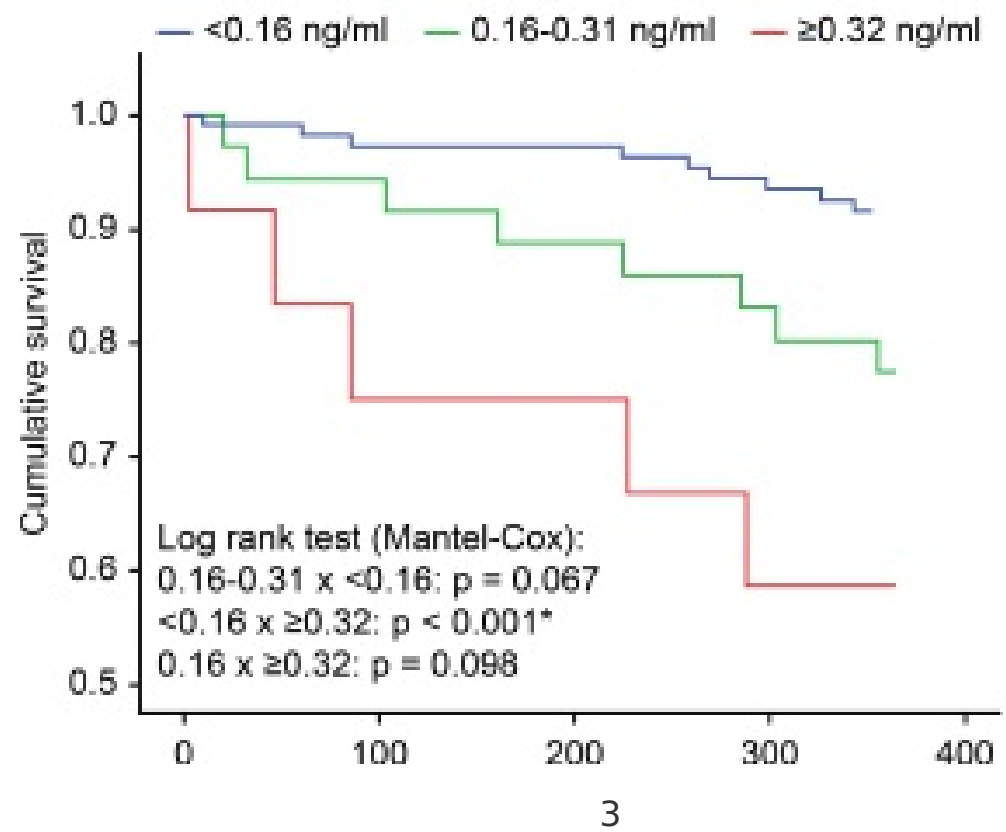




\section{Postoperative period (days)}

\section{Figure 1}

Survival within one year in patients who had increased troponin I levels during the postoperative period of lung resection surgery according to the time when the elevation was

detected: A) Immediate postoperative time, B) First postoperative day, C) Second postoperative day. $p=0.05$. 